\title{
El Observatorio de Turismo, base para el Centro de Articulación Productiva de Turismo de Quintana Roo
}

DOI: 10.22403/UQROOMX/TYP05/12

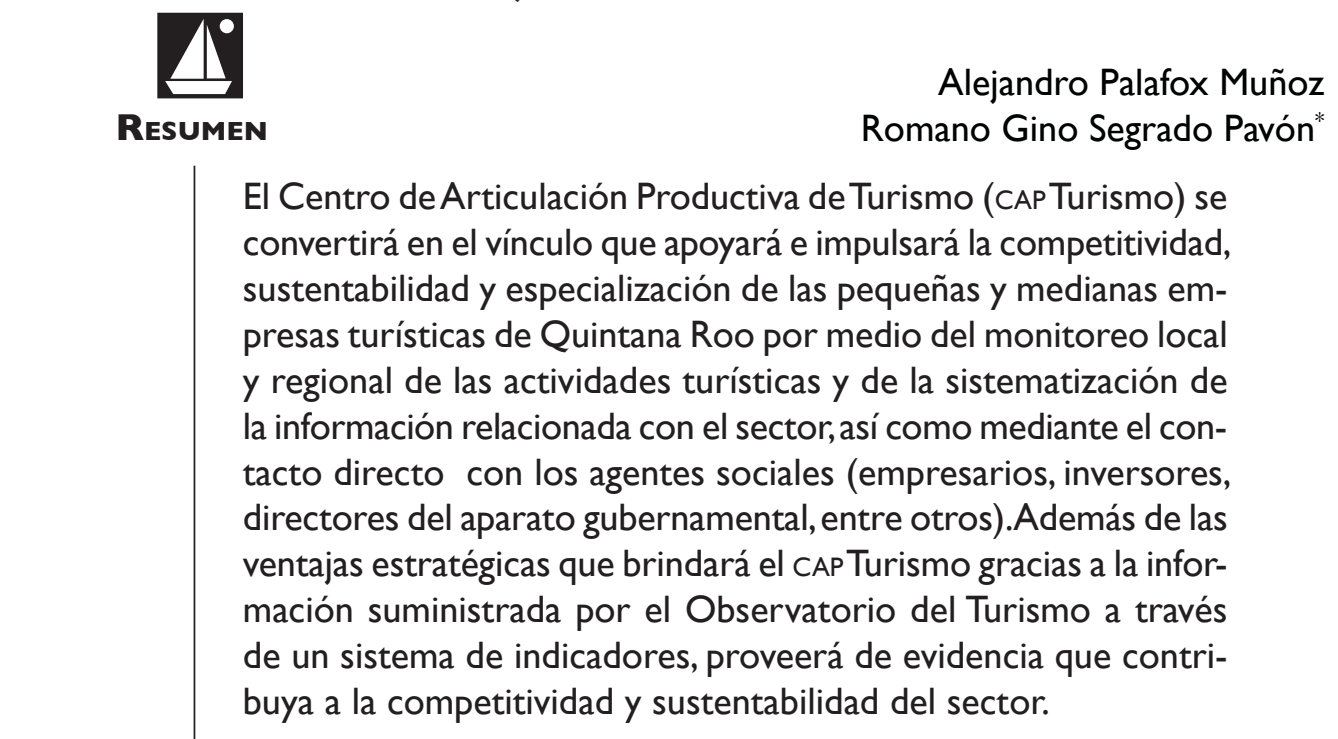

Palabras | Quintana Roo, Cap, Turismo, Observatorio del Turismo.

CLAVE

*Universidad de Quintana Roo, Cuerpo Académico de Turismo / palafox@uqroo.mx / romano@uqroo.mx 
El Observatorio de Turismo

\section{EI contexto}

El acceso a la información "es un derecho que todo ciudadano tiene" (JICA y CNREE, 2006: 7); pese a ello, la escasez de estadísticas confiables, su dispersión en diferentes entidades públicas y privadas, y la falta de actualización de la información, impiden el establecimiento de un sistema que contenga los datos necesarios para el estudio y resolución de problemas del sector turismo.

Los sistemas de información son un instrumento que coadyuva a medir y monitorear los hechos sociales, económicos y ambientales, para la toma de decisiones en el establecimiento de políticas de desarrollo, la asignación de recursos y la evaluación de las actividades que se han llevado a cabo.

En México, el turismo contribuye significativamente al producto interno bruto del país (véase cuadro I). Desde 1993, la aportación de la actividad turística al PIB ha permanecido más o menos estable; sin embargo, en el año 2002 tuvo una baja de cuatro décimas porcentuales, que no se han recuperado y que incluso han seguido disminuyendo una décima porcentual de forma sostenida en los últimos cuatro años, llegando a reducir su participación en siete décimas.

Cuadro I. Contribución del tURISMo al pib nacional

\begin{tabular}{cccccccccccccc}
\hline Aก̃o & 1993 & 1994 & 1995 & 1996 & 1997 & 1998 & 1999 & 2000 & 2001 & 2002 & 2003 & 2004 & 2005 \\
$\%$ & 8.1 & 8.2 & 8.4 & 8.2 & 8.2 & 8.3 & 8.4 & 8.4 & 8.4 & 8.0 & 7.9 & 7.8 & 7.7 \\
\hline
\end{tabular}

Fuente: Compendio estadístico del turismo en México 2006.

El turismo en el estado de Quintana Roo aporta $80 \%$ del PIB estatal (Gobierno del Estado de Quintana Roo, 2006:54) y capta $27.4 \%$ de divisas por concepto de turismo, lo que se traduce en 3235.03 millones de dólares, según datos de la Secretaría de Turismo del Estado de Quintana Roo (2007); sin embargo, el desarrollo de la actividad está orientado al norte del estado en los destinos de Cancún, la Riviera Maya (Puerto Morelos, Playa del Carmen y Akumal y Tulum), y Cozumel, y un incipiente desarrollo al sur con el megaproyecto de Costa Maya (Mahahual).

Ante este panorama, el Cuerpo Académico de Turismo (CAT) de la Universidad de Quintana Roo determinó crear el Observatorio del Turismo para elaborar proyectos de investigación científica relacionados con la actividad turística, buscando resolver las necesidades de la sociedad. Lo anterior se realizará 
midiendo y monitoreando la sustentabilidad de los destinos turísticos de la entidad, recurriendo a un sistema de indicadores de turismo sustentable que apoyen la toma de decisiones de los actores del turismo.

\section{El turismo en Quintana Roo}

La economía quintanarroense está basada en el turismo, que es considerado el eje del desarrollo estatal. En 2006 llegaron a la entidad 9.9 millones de visitantes, de los cuales $59 \%$ eran turistas, $34.3 \%$ pasajeros de crucero y $6 \%$ excursionistas fronterizos (México-Belice). Con relación a los últimos siete años (véase gráfica I), el porcentaje de captación de divisas ha ido disminuyendo. En el año 2000, Quintana Roo recibía 37\% de las divisas nacionales por concepto de turismo, pero la caída en este rubro ha sido consistente $y$, como resultado, para 2006, el porcentaje de captación fue $27.7 \%$.

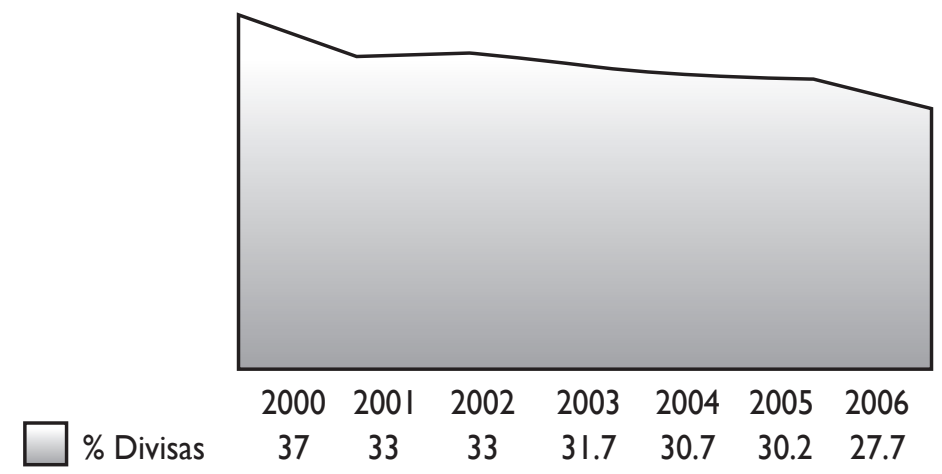

Fuente: Elaboración propia con datos de Sedetur (2007).

Gráfica I. CAPTACIÓN de diVISAS A NIVEL NACIONAL

Por otra parte, en los últimos siete años, el estado ha recibido la visita de 62675 I 58 viajeros en diversos segmentos: $5.2 \%$ excursionista fronterizo (México-Belice), $31.3 \%$ pasajeros de crucero (Cozumel, Mahahual, Playa del Carmen y Cancún) y $63.3 \%$ turistas (véase gráfica 2). Es pertinente comentar que el ascenso en la captación de turistas fue considerable $(25.6 \%)$ hasta el año 2004 , ya que a partir de entonces, se ha registrado una caída de hasta $11.7 \%$. 
El Observatorio de Turismo

Lo mismo ha ocurrido con el turismo de cruceros, que creció $42.3 \%$ y luego disminuyó $4.2 \%$.A pesar de lo anterior, la tendencia del turismo fronterizo ha ido a la alza en los últimos tres años.

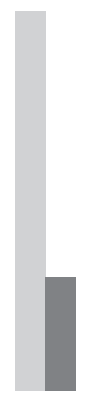

2000

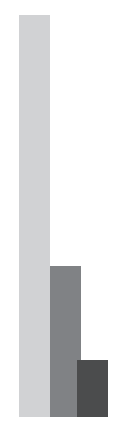

2001

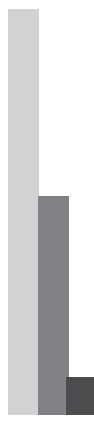

2002

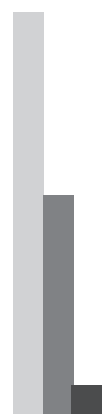

2003

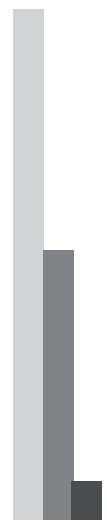

2004

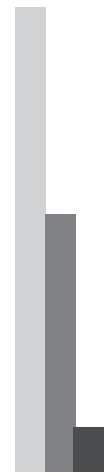

2005

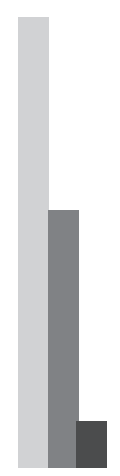

2006

- Turistas $\quad 50028475294514 \quad 5350865 \quad 5312438 \quad 6726940 \quad 6112670 \quad 5935316$

•Cruceristas I $504604 \quad$ | $9938462893495 \quad 291075$ | 355345733884523403417

$\begin{array}{lllllll}\text { G Fronterizos } & 720071 & 492028 & 394954 & 522798 & 566981 & 614714\end{array}$

Fuente: Elaboración propia con datos de Sedetur (2007).

Gráfica 2.Afluencia de turistas en Quintana Roo (2000-2006)

La infraestructura hotelera ha sido pieza fundamental en el desarrollo de la actividad turística en Quintana Roo, que ha logrado ser la entidad con mayor capacidad de alojamiento en México. En este tenor, el Gobierno del Estado de Quintana Roo (2005) menciona que la oferta hotelera del estado en el año 2004 equivalía a I2\% del total del país, con 763 hoteles y 59497 cuartos. En 2006 y después de las afectaciones del huracán Wilma, la entidad contaba con 63322 cuartos repartidos en 722 hoteles, con una ocupación hotelera de 67.13\%.

Los datos señalan un descenso en la llegada de turistas y en la participación en la economía nacional, lo cual se puede inferir por la carente diversidad de productos y servicios turísticos, dado que la mayoría de éstos están dirigidos al segmento de sol y playa, con mínimas diferencias en costos y amenidades, o bien, 
como señala Butler (1980), el ciclo de vida del destino turístico entró a su etapa de decadencia, por lo que es necesario comenzar su recuperación, diversificando la oferta basada en las necesidades del mercado, para ello es necesaria la recopilación, tratamiento, sistematización, análisis e interpretación de información obtenida mediante indicadores, que "son una herramienta básica que permite observar y prevenir conflictos” (Frausto et al., 2002: I27), además de ser un elemento importante para la toma de decisiones y elaboración de políticas.

Aun cuando el Plan Gran Visión 2000-2025 del estado (Gobierno del Estado de Quintana Roo, 200I), determina la diversificación de la oferta, la política turística continua promoviendo las actividades de sol y playa, lo que se ratifica con el último megaproyecto turístico promovido: Costa Maya, destino que cuenta con un muelle para la recepción de tres cruceros, y donde durante 2006 arribaron 348 barcos, con un total de 81 I 287 pasajeros, $19.8 \%$ más que los 12 meses previos.

El estado enfrenta grandes retos en materia turística para los años venideros, por ello es importante que los actuales proyectos y modelos de desarrollo turístico para Quintana Roo se apeguen al desarrollo sustentable de la actividad, y que realmente se diversifique la oferta y el fomento de productos turísticos acordes con las necesidades del cliente y en busca del desarrollo regional, que integre las zonas marginadas y eleve la calidad de vida de los quintanarroenses.

\section{El Centro de Articulación Productiva de Turismo (CAP Turismo)}

El Fondo para las Pequeñas y Medianas Empresas de la Secretaría de Economía define un CAP como la institución que tiene por objetivo la generación de más y mejores empleos, y de más y mejores empresas para el desarrollo sustentable y competitivo del país. Su finalidad es apoyar el desarrollo y la competitividad de las micro, pequeñas y medianas empresas (mipymes), así como integrar, articular y fortalecer las cadenas productivas y redes de valor.

Su objetivo es apoyar a las mipymes mexicanas mediante la prestación de servicios de consultoría y asesoría, dirigiendo sus acciones a la articulación de los procesos productivos, el fortalecimiento de empresas y la vinculación entre oferta y demanda. 
El Observatorio de Turismo

Las principales funciones del CAP son:

- Generar empresas competitivas, integrar cadenas productivas inteligentes, elevar el conocimiento empresarial, transferir nueva tecnología, dotar de mayor capacidad humana y técnica, generar mayor volumen de negocio, y propiciar certidumbre de mercado.

Dentro de los propósitos del gobierno de Quintana Roo se encuentra el convertir al turismo en una prioridad, por ello la Universidad de Quintana Roo y la Secretaría de Desarrollo Económico del estado, así como la Secretaría de Economía se unieron para crear el CAPTurismo, el cual es entendido como un órgano de apoyo para el fortalecimiento del sector turístico de la entidad, enfocado al desarrollo empresarial y humano.

Tomando en cuenta las líneas estratégicas del Plan Nacional de Desarrollo 2007-20 I 2, el Plan Estatal de Desarrollo 2005-20 I I, el Plan Gran Visión 20002025, y el Programa de Desarrollo Turístico del Sur se genera el modelo del CAP Turismo, integrado por dos ramas, consideradas como líneas estratégicas:

- Inteligencia competitiva

- Desarrollo del capital humano

Estas líneas permiten al CAP contribuir con el sector turístico al identificar las necesidades existentes, así como las oportunidades de desarrollo, a través de la generación y monitoreo de indicadores en un contexto de desarrollo sustentable, para la toma de decisiones.

Shrivastava y Grant (1985) definen la inteligencia competitiva como el sistema de aprendizaje sobre las capacidades y comportamientos de los competidores actuales y potenciales con el objeto de ayudar a los responsables en la toma de decisión estratégica. Asimismo la literatura sobre estrategia y marketing a menudo precisa que la inteligencia competitiva es un sistema de alarma temprana, que localiza y organiza colectivamente los esfuerzos individuales sobre acontecimientos que pueden ser relevantes para la empresa (Palop y Vicente, 1999).

Para tal efecto, el CAP Turismo desarrolla tres proyectos:

I) Observatorio del Turismo

2) Centro de Innovación y Desarrollo de Turismo

3) Programa de Gestión Estratégica de Destinos Turísticos 


\section{El Observatorio del Turismo de la}

\section{Universidad de Quintana Roo}

Sin duda, la actividad económica más importante del estado de Quintana Roo es el turismo y, como se ha advertido, su participación en la economía nacional ha ido en descenso. Por ello, la Universidad de Quintana Roo, mediante su Cuerpo Académico de Turismo y comprometida con la investigación turística del entorno nacional, regional y local, establece un organismo que pretende generar información confiable sobre la actividad turística para la toma de decisiones de sus actores sociales: el Observatorio del Turismo (véase figura I).

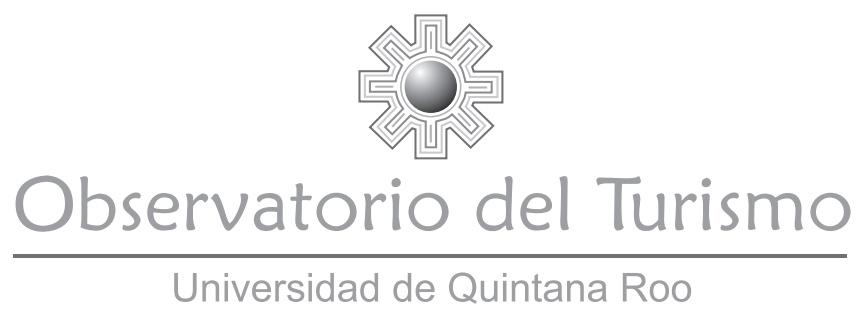

Fuente: Observatorio del Turismo.

Figura I. Logotipo del Observatorio del Turismo

El Observatorio del Turismo llevará a cabo su quehacer a través de las siguientes acciones:

I. Monitoreando y midiendo la sustentabilidad de los destinos turísticos mexicanos, en particular del estado de Quintana Roo.

2. Dotando de información relevante para apoyar la toma de decisiones de los actores públicos y privados.

3. Realizando investigaciones especializadas sobre asuntos de interés público y privado vinculados con el turismo.

4. Poniendo en marcha un sistema de indicadores de turismo sustentable que permitan prever los resultados de las políticas e iniciativas de índole turística. 
El Observatorio de Turismo

Lo anterior se logrará gracias a:

- La creación y actualización de una base de datos con indicadores seleccionados (UN-WTO/GEO Ciudades/UN-Habitat)

- Proyectos específicos

- Presentación de resultados en foros académicos

- Reportes a las instituciones públicas y privadas

- Artículos de los investigadores del Observatorio

- Tesis de licenciatura, maestría y doctorado

En este sentido, los proyectos realizados serán supervisados y operados por especialistas de diferentes disciplinas:Turismo, Mercadotecnia, Geografía, Administración, Economía, Psicología, Comunicación, Biología y Sociología, entre otras. Estos expertos se encuentran adscritos a la División de Desarrollo Sustentable de la Universidad de Quintana Roo,y forman el Cuerpo Académico de Turismo de la institución.

\section{Conclusiones}

La creación del Observatorio del Turismo da continuidad a las tendencias internacionales referentes a este tipo de organismos, interesados en generar y concentrar información para ofrecer indicadores que coadyuven en la toma de decisiones y, en este caso concreto para el Centro de Articulación Productiva de Turismo del estado de Quintana Roo, el cual tiene como finalidad la búsqueda de alternativas de negocio para la generación de riqueza mediante el impulso de medianas y pequeñas empresas.

Por otro lado, el Centro de Articulación Productiva de Turismo del estado de Quintana Roo podrá constituirse como un ente especializado de consultoría para la creación y desarrollo de negocios turísticos; en tanto el Observatorio del Turismo colaborará produciendo información que facilite y sustente una mejor toma de decisiones de negocios.

Sin embargo, el Observatorio también realizará proyectos de investigación conjunta; por lo cual la Universidad de Quintana Roo invita a las instituciones académicas a formar parte del Observatorio del Turismo, que se ha gestado en el seno del Cuerpo Académico de Turismo, y cuyos integrantes forman parte 
de la Red de Cuerpos Académicos en Desarrollo y Turismo (ReCADyT), Red de Investigadores y Centros de Investigación en Turismo (RICIT), así como de la Asociación Mexicana de Investigación Turística (AMIT), que a través de alianzas de cooperación académica reconocen la necesidad de contribuir al desarrollo turístico y sustentable del país.

El apoyo financiero es imperativo para el buen desempeño del Observatorio, y en un principio se recibirán fondos de los gobiernos estatal y federal, a través del CAP Turismo, para iniciar los trabajos de recuperación de información turística para el sistema de indicadores de turismo sustentable que desarrolla el Observatorio del Turismo.

\section{Agradecimientos}

A la Universidad de Quintana Roo por el apoyo brindado al Cuerpo Académico de Turismo y en especial a la División de Desarrollo Sustentable por el respaldo en la creación del Observatorio del Turismo. También, al Centro Emprendedor de Negocios de la institución por su invitación a participar en el Centro de Articulación Productiva de Turismo, el cual sirve de intermediario entre las instancias federales y estatales para la obtención de recursos económicos.

\section{FUENTES CONSULTADAS}

Butler, R. (1980)."The concept of tourist area cycle of evolution: implications for management of resources”. Canadian Geographer, XXIV (I), 5- 12.

Frausto Martínez, O.,S. Giese y G. Chalé (2002). “Indicadores ambientales de turismo sustentable para el Caribe y Centroamérica: Isla de Cozumel, México" en: Alberto Pereira, lan Boxill y Johannes Maerk (coords.)Turismo, desarrollo y recursos naturales en el Caribe. México: Plaza y Valdés / Universidad de Quintana Roo / University of West Indies, 264 p.

Gobierno del Estado de Quintana Roo (200I). Plan Gran Visión 2000-2025. México: Gobierno del Estado de Quintana Roo.

(2005). Turismo: los resultados de una gestión. México: Gobierno del Estado de Quintana Roo.

(2006). Primer Informe de Gobierno. México: Gobierno del Estado de Quintana Roo. 
El Observatorio de Turismo

JICA y CNREE (2006). Construyendo el Observatorio de la Discapacidad. Costa Rica: Agencia de Cooperación Internacional de Japón/Consejo Nacional de Rehabilitación y Educación Especial.

Palop, F. y J. M. Vicente (1999). Vigilancia tecnológica e inteligencia competitiva. Su potencial para la empresa española. Disponible en http://www. navactiva.com/web/es/descaras/pdf/avtec/potencial_vtec.pdf, [2007, 28 de junio].

Sectur (2006). Compendio estadístico del turismo en México 2006. México: Secretaría de Turismo.

Sedetur (2007). “Indicadores turísticos" [en línea]. Secretaría de Turismo del Estado de Quintana Roo. Disponible en: http://sedetur.qroo.gob.mx/ estadisticas/estadisticas.php

Sen, A. (1998). “Capital humano y capacidad humana”. Cuadernos de Economía, XVII (29), 67-72.

Shrivastava, P.y J. Grant ( 1985$)$."Empirically derived models on strategic decision making process”. Strategic Management Journal, 6 (2), 97-I I 3. 